(1)

CrossMark

\title{
TBX4 syndrome: a systemic disease highlighted by pulmonary arterial hypertension in its most severe form
}

\author{
Eric D. Austin $\mathbb{0}^{1}$ and C. Gregory Elliott ${ }^{2}$ \\ Affiliations: ${ }^{1}$ Dept of Pediatrics, Vanderbilt University Medical Center, Nashville, TN, USA. ${ }^{2}$ Dept of Medicine, \\ Intermountain Medical Center and the University of Utah, Murray, UT, USA. \\ Correspondence: Eric D. Austin, Dept of Pediatrics, Division of Pulmonary, Allergy, and Immunology \\ Medicine, DD-2205 Medical Center North, Vanderbilt University School of Medicine, Nashville, TN, 37232-
} 2578, USA. E-mail: eric.austindavumc.org

@ERSpublications

Thoré and co-workers add to our understanding of TBX4-associated pulmonary vascular disease as a precapillary form of pulmonary hypertension (PH), showing that TBX4 mutations may also cause multisystem anomalies concurrent with, or independent of, $\mathbf{P H}$ https://bit.ly/2yIqb9v

Cite this article as: Austin ED, Elliott CG. TBX4 syndrome: a systemic disease highlighted by pulmonary arterial hypertension in its most severe form. Eur Respir J 2020; 55: 2000585 [https://doi.org/10.1183/ 13993003.00585-2020].

\begin{abstract}
Sometimes we miss the "ah-ha" moment, or, at least require multiple reminders to pay attention. This may have been the case for many of us in the pulmonary vascular field regarding rare variants (mutations) in the gene T-box transcription factor 4 (TBX4) and pulmonary hypertension (PH). In 2004, investigators in the Netherlands published the first description of humans with mutations in the T-box transcription factor 4 (TBX4) gene. While an interesting manuscript, it undoubtedly created no ripple in the pulmonary vascular disease field, as it described a cohort of individuals with small patella syndrome (SPS) without known cardiopulmonary abnormalities, consistent with prior animal model studies [1,2]. At that time, SPS was known as a rare autosomal-dominant condition characterised by skeletal dysplasia, including irregular development of the patella and additional anomalies involving the limbs and pelvis. Subsequently, in 2010, geneticists expanded our understanding of the phenotype of subjects with mutations in TBX4 (and perhaps other loci nearby, including TBX2), by describing paediatric cases with a more broadly syndromic condition characterised by variable expression of anomalies, such as skeletal dysplasias (including hand and foot irregularities), developmental delay, hearing loss, congenital heart defects (e.g. patent ductus arteriosus, atrial septal defect, aortic valve defects), and $\mathrm{PH}$ (no specific $\mathrm{PH}-$ related data was provided) [3, 4]. Again, the pulmonary vascular field, current authors included, failed to take notice. Then, in 2013, Kerstjens-Frederikse et al. [5], from a paediatric PH specialty centre in the Netherlands, described variations in TBX4 and surrounding loci in six out of 20 paediatric pulmonary arterial hypertension (PAH) cases associated with syndromic anomalies similar to those previously described in TBX4 mutants; overall, their data suggested that TBX4 mutations contribute to the PAH condition in paediatrics. Yet, many in our field retained the impression that TBX4 mutations were more prominent among children with highly "syndromic" features, and thus associated with a niche subgroup of $\mathrm{PH}$ in children.
\end{abstract}

In hindsight, these important work products implicating TBX4 in the pathogenesis of PH set the stage for the subsequent emergence of impactful findings elucidating a notable prevalence of TBX4 mutations 
among individuals previously felt to have a very "PH-specific phenotype" with familial (FPAH) or idiopathic PAH (IPAH). The gaze of the pulmonary vascular field has shifted dramatically to TBX4 over the past 4 years, starting with a Spanish report of heterozygous TBX4 mutations in three out of $136(2.2 \%)$ adults diagnosed with IPAH [6]. Shortly thereafter, ZHU et al. [7] reported a US cohort of 412 individuals diagnosed with FPAH or IPAH in which deleterious heterozygous mutations in TBX4 were discovered, with both paediatric-onset $(12 / 155 ; 7.7 \%)$ and adult-onset $(1 / 257 ; 0.4 \%)$ cases. Subsequently, TBX4 was the second most common $(23 / 2572 ; 0.89 \%)$ mutation identified in PAH cases reported from the US PAH Biobank, with 12/266 (4.5\%) paediatric-onset and 11/2345 (0.47\%) adult-onset cases. The PAH Biobank cohort included all subtypes of PAH (i.e. it was not restricted to FPAH and IPAH cases) [8]. Of the 23 cases with TBX4 mutations in the PAH Biobank, two (8.7\%) had an associated connective tissue disease (one paediatric and one adult-onset case) while three (13.0\%) had congenital heart disease-associated PAH (two paediatric and one adult-onset) [8]. Deeper phenotypic information regarding skeletal or other features was not readily available from the PAH Biobank publication.

These studies and several others suggest that heterozygous TBX4 mutations are rare but detectable among patients with what we typically consider as group $1 \mathrm{PH}(\mathrm{PAH})$, according to the most recent Nice Classification System [9-11]. Yet, not all of the subjects with $\mathrm{PH}$ and heterozygous TBX4 mutations fit neatly in the group 1 classification. In addition to the reports described above, a growing body of literature describes profound defects of lung development associated with heterozygous TBX4 mutations (or large gene deletions encompassing TBX4), including death during infancy due to acinar dysplasia, congenital alveolar dysplasia, and pulmonary hypoplasia [12-15]. This is not surprising, given the important role in development, including but not limited to the lung and skeletal system, of TBX4 and related molecular pathway members [16]. Consistent with this recognition, children with severe persistent precapillary $\mathrm{PH}$ diagnosed during infancy with detectable anomalies such as congenital heart disease, skeletal defects, and developmental disabilities are now recognised to populate many of our paediatric PH programmes [17].

In the current issue of the European Respiratory Journal, THORE et al. [18], from the French National Registry, report their experience in the care of 20 patients from 17 families known to transmit a variety of heterozygous mutations in TBX4. The authors provide comprehensive phenotypic and long-term outcome data on these $\mathrm{PH}$ subjects with a precapillary form of $\mathrm{PH}$ consistent with severe PAH. Of the 448 family probands with $\mathrm{PAH}$ studied, $6 \%$ of paediatric-onset, versus $3 \%$ of adult-onset, had TBX4 mutations. TBX4-associated PAH in France demonstrated a female predominance and a bimodal age distribution, with presentation typically either during childhood or after age 40 years [8]. The majority of subjects had skeletal abnormalities including a high SPS penetrance, while congenital heart disease was less prevalent (15\% of cases). The degree of detectable lung irregularities among these patients otherwise diagnosed with PAH was striking, including obstructive or restrictive abnormalities of pulmonary function, significant reductions in diffusing capacity of the lung for carbon monoxide corrected for haemoglobin and structural changes in the lungs. The high degree of bronchial and/or tracheal diverticula of the large airways, as well as parenchymal lung lesions, were consistent with concerns that disruptions of TBX4 signalling may result in profound or more subtle developmental lung lesions.

The non-vascular lung disease among adult heterozygous TBX4 mutation carriers highlighted by the French National Registry is consistent with recent smaller reports of adult PAH patients [19], including in this issue of the European Respiratory Journal. JANSEN et al. [20] in the Netherlands described one paediatric-onset and four adult-onset PAH cases with TBX4 mutations, all among females, as well as three male TBX4 mutation carriers without PAH. Every subject had skeletal anomalies as well as tracheal and/or bronchial diverticulosis, regardless of $\mathrm{PAH}$ status. Among those with $\mathrm{PAH}$, additional functional and imaging-based irregularities were detected, similar to TBX4 mutation carriers in the French National Registry.

Taken together, there is now ample data from both paediatric- and adult-onset cases to demonstrate that TBX4-associated pulmonary vascular disease is haemodynamically a precapillary PH consistent with PAH. However, the story is more complicated. We propose that those subjects with deleterious heterozygous TBX4 mutations or TBX4-containing deletions have "TBX4 syndrome", which, while heterogenous, is characterised by precapillary PH in its most severe form. The reason that "TBX4 syndrome" presents with profound perinatal cardiopulmonary disease for some, SPS alone in others, or a combination of features that may include PAH remains unclear. The variability in presentation likely has to do with undetermined genomic, transcriptomic, epigenomic, or other factors that shape lung development, as well as susceptibility to injury and environmental influences. As with BMPR2-associated and other forms of PAH, the female predominance suggests sex-related factors contribute to PAH pathogenesis in TBX4 mutants, but further work is needed. What is clear is that the prevalence of TBX4 syndrome in our PH clinics is not trivial; and, even prior to genetic testing, careful examination for skeletal abnormalities, airway diverticulosis, abnormal pulmonary function test results, or the presence of atrial septal and other congenital heart defects, should heighten suspicion of heritable TBX4 syndrome. 
THORÉ et al. [18], and other recent studies, have contributed important advances to our understanding of TBX4 syndrome. Future studies are needed to explore the molecular and other features of TBX4 signalling, especially as it relates to the pulmonary vasculature and phenotypic variations. Given its relatively rarity, international collaborations will be important to build sufficient numbers of patients to support evaluation of TBX4 syndrome in more depth.

Finally, the placement of TBX4 syndrome within the $\mathrm{PH}$ diagnostic classification demands careful consideration. This issue also highlights a broader challenge for $\mathrm{PH}$ specialists: as we learn more about phenotypic diversity in $\mathrm{PH}$ conditions, allocating our patients into discrete $\mathrm{PH}$ classification groups becomes more difficult. Is $\mathrm{PH}$ in TBX4 syndrome a developmental lung disease (Nice group 3), or a heritable form of $\mathrm{PH}$ most consistent with group I (PAH), or both? Regardless, the emergence of TBX4 syndrome as a recognised cause of $\mathrm{PH}$ is further evidence that $\mathrm{PH}$ may occur as a component of many, at times heritable, different conditions.

Conflict of interest: C.G. Elliott has nothing to disclose. E.D. Austin has nothing to disclose.

Support statement: Funding was provided by NIH R01 HL 134802 (E.D. Austin). Funding information for this article has been deposited with the Crossref Funder Registry.

\section{References}

1 Bongers EM, Duijf PH, van Beersum SE, et al. Mutations in the human TBX4 gene cause small patella syndrome. Am J Hum Genet 2004; 74: 1239-1248.

2 Naiche LA, Papaioannou VE. Loss of Tbx4 blocks hindlimb development and affects vascularization and fusion of the allantois. Development 2003; 130: 2681-2693.

3 Ballif BC, Theisen A, Rosenfeld JA, et al. Identification of a recurrent microdeletion at 17q23.1q23.2 flanked by segmental duplications associated with heart defects and limb abnormalities. Am J Hum Genet 2010; 86: 454-461.

4 Nimmakayalu M, Major H, Sheffield V, et al. Microdeletion of 17q22q23.2 encompassing TBX2 and TBX4 in a patient with congenital microcephaly, thyroid duct cyst, sensorineural hearing loss, and pulmonary hypertension. Am J Med Genet A 2011; 155A: 418-423.

5 Kerstjens-Frederikse WS, Bongers EM, Roofthooft MT, et al. TBX4 mutations (small patella syndrome) are associated with childhood-onset pulmonary arterial hypertension. J Med Genet 2013; 50: 500-506.

6 Navas P, Tenorio J, Quezada CA, et al. Molecular analysis of BMPR2, TBX4, and KCNK3 and genotypephenotype correlations in Spanish patients and families with idiopathic and hereditary pulmonary arterial hypertension. Rev Esp Cardiol (Engl Ed) 2016; 69: 1011-1019.

7 Zhu N, Gonzaga-Jauregui C, Welch CL, et al. Exome sequencing in children with pulmonary arterial hypertension demonstrates differences compared with adults. Circ Genom Precis Med 2018; 11: e001887.

8 Zhu N, Pauciulo MW, Welch CL, et al. Novel risk genes and mechanisms implicated by exome sequencing of 2572 individuals with pulmonary arterial hypertension. Genome Med 2019; 11: 69.

9 Simonneau G, Montani D, Celermajer DS, et al. Haemodynamic definitions and updated clinical classification of pulmonary hypertension. Eur Respir J 2019; 53: 1801913.

10 Eyries M, Montani D, Nadaud S, et al. Widening the landscape of heritable pulmonary hypertension mutations in paediatric and adult cases. Eur Respir J 2019; 53: 1801371.

11 Levy M, Eyries M, Szezepanski I, et al. Genetic analyses in a cohort of children with pulmonary hypertension. Eur Respir J 2016; 48: 1118-1126.

12 Karolak JA, Vincent M, Deutsch G, et al. Complex compound inheritance of lethal lung developmental disorders due to disruption of the TBX-FGF pathway. Am J Hum Genet 2019; 104: 213-228.

13 Karolak JA, Szafranski P, Kilner D, et al. Heterozygous CTNNB1 and TBX4 variants in a patient with abnormal lung growth, pulmonary hypertension, microcephaly, and spasticity. Clin Genet 2019; 96: 366-370.

14 German K, Deutsch GH, Freed AS, et al. Identification of a deletion containing TBX4 in a neonate with acinar dysplasia by rapid exome sequencing. Am J Med Genet A 2019; 179: 842-845.

15 Suhrie K, Pajor NM, Ahlfeld SK, et al. Neonatal lung disease associated with TBX4 mutations. J Pediatr 2019; 206 : 286-292.

16 Vincent M, Karolak JA, Deutsch G, et al. Clinical, histopathological, and molecular diagnostics in lethal lung developmental disorders. Am J Respir Crit Care Med 2019; 200: 1093-1101.

17 Galambos C, Mullen MP, Shieh JT, et al. Phenotype characterisation of TBX4 mutation and deletion carriers with neonatal and paediatric pulmonary hypertension. Eur Respir J 2019; 54: 1801965.

18 Thoré P, Girerd B, Jaïs X, et al. Phenotype and outcome of pulmonary arterial hypertension patients carrying a TBX4 mutation. Eur Respir J 2020; 55: 1902340.

19 Maurac A, Lardenois E, Eyries M, et al. T-box protein 4 mutation causing pulmonary arterial hypertension and lung disease. Eur Respir J 2019; 54: 1900388.

20 Jansen SMA, van den Heuvel L, Meijboom LJ, et al. Correspondence regarding "T-box protein 4 mutation causing pulmonary arterial hypertension and lung disease": a single-centre case series. Eur Respir J 2020; 55: 1902272. 